\title{
Antibacterial Activity of Fusidic Acid and Sodium Fusidate Nanoparticles Incorporated in Pine Oil Nanoemulgel
}

This article was published in the following Dove Press journal: International Journal of Nanomedicine

\author{
Ahmad M Eid (iD \\ Ibraheem Istateyeh \\ Noura Salhi \\ Thaer Istateyeh
}

Department of Pharmacy, Faculty of Medicine and Health Sciences, An-Najah National University, Nablus, Palestine
Correspondence: Ahmad M Eid Pharmaceutical Chemistry and Technology Division, Department of Pharmacy, Faculty of Medicine and Health Sciences, An-Najah National University, P.O. Box 7, Nablus, Palestine Email ahmadeid@najah.edu
Purpose: Fusidic acid (FA) and sodium fusidate (SF) have problems in their skin penetration and stability resulting in a reduction in their potency; therefore, the objective of this study was to develop FA and SF nanoemulgels to improve the antibacterial activity of the drugs.

Methods: FA and SF nanoemulgel formulations were prepared by the incorporation of FA and SF nanoemulsions with Carbopol hydrogel. First, the drugs were screened for their solubility in different oils and surfactants to choose the suitable oil and surfactants for the drugs, and then the drug nanoemulsion formulations were prepared by a self-nanoemulsifying technique using Tween 80, Span 20 and pine oil. The drug nanoemulgels were evaluated for their particle size, polydispersity index (PDI), rheological behaviour, drug release and anti-microbial activity.

Results: Based on the solubility test, pine oil was the best solubilising oil for both drugs, Tween 80 and Span 20 showed the highest solubilising ability for both the drugs among the surfactants; therefore, they were chosen as surfactant and co-surfactant, respectively. The optimum self-nanoemulsifying formulations showed a particle size for fusidic acid and Sodium fusidate of $140.58 \mathrm{~nm}$ and $151.86 \mathrm{~nm}$ respectively, and both showed a low PDI below 0.3. After incorporating both drug SNEDDS formulations with Carbopol at different concentrations, the results of the drugs particle size and PDI showed no significant difference. The zeta potential results for both drugs nanoemulgels showed a negative potential with more than $30 \mathrm{mV}$. All nanoemulgel formulations showed pseudo-plastic behaviour with the highest release pattern at $0.4 \%$ Carbopol. The antibacterial activity of both drug nanoemulgel formulations showed superiority over the market product.

Conclusion: Nanoemulgel is a promising delivery system for FA and SF that helps in improving the stability and antibacterial activities of the drugs.

Keywords: fusidic acid, sodium fusidate, nanoemulgel, pine oil, antibacterial activity

\section{Introduction}

Nanotechnology is a field focused on creating materials with new functional and beneficial characteristics through controlling of the structurelsequence of material at the nanometre size; ${ }^{1,2}$ the field is also concerned with the development of nanotechnology support improvements for new treatments for various diseases. ${ }^{3}$ Nanoemulsion is a novel delivery system, which is one of the growing technologies for drugs and lipophilic materials. Nanoemulsion is named due to its droplet sizes which range between $20 \mathrm{~nm}$ and $200 \mathrm{~nm}$. Nanoemulsions have been used in the pharmaceutical field as a drug delivery system through various systemic routes such as oral, topical and parenteral nutrition. ${ }^{4}$ 
The technique of nano-emulsification has come from various modifications and improvements in the limitation routes faced through the drug delivery processes. ${ }^{5}$ The term comes from the incorporation of nanoemulsion and hydrogel. Obtaining better stability of a nanoemulsion due to the gelling system by the reduction of the surface and interfacial tension and a better topical administration by enhancing the viscosity of the aqueous. ${ }^{6,7}$ Moreover, nanoemulgel, which basically includes gel, shows an improvement in thixotropic properties, non-greasy, easily spreadable, easy to remove, not staining, water-soluble, emollient, longer shelf life and an agreeable appearance. ${ }^{6,8}$ The formulation eliminates the difficulty of spreading and supports patient compliance in administration since is not sticky. ${ }^{9}$

Antimicrobial resistance is considered a major public health problem with the second-leading cause of deaths worldwide. World Health Organization (WHO) expressed serious concern regarding an increase in antimicrobial resistance rates in recent years. ${ }^{10}$ This leads to the need of new strategies to either develop of new antibiotics or enhancement of the effectiveness of existing antibiotics. Therefore, several studies provide an overview on the management of microbial infection and antimicrobial resistance. Nanostructured materials are one of these strategies which are being developed to overcome some types of bacterial resistance. Therefore, Nanoparticles may offer a promising solution as they can not only combat bacteria themselves, but can also act as carriers for antibiotics by circumvent drug resistance mechanisms in bacteria and assist in the delivery of novel drugs. ${ }^{11,12}$

Fusidic Acid (FA) is derived from fusidium coccineum, which is a fungus that was originally isolated in 1962 from monkey faeces. ${ }^{13,14}$ Fusidic Acid (FA) has a bacteriostatic activity and is available in many formulations such as oral, topical, and parenteral formulation for various treatments for infection. In contrast, many adverse effects are related to oral and parenteral routes, including gastrointestinal and liver upset, hepatotoxicity, diarrheal, phlebitis and rhabdomyolysis. This leads to a reduction in its therapeutic efficacy at the target site due to systematic distribution and absorption of FA to different organs of the body. ${ }^{15,16}$ There are many problems linked with topical routes such as poor spreadability and dermal penetration, local irritation, and sub-optimal efficacy. ${ }^{17-20}$

Sodium fusidate which inhibits bacterial protein synthesis is Staphylococci, including penicillinase producing and methicillin resistant strains. ${ }^{21}$ With the exception of the Neisseriae and Bacteroides spp., Gram-negative bacteria are insensitive to sodium fusidate. Gram-positive organisms highly sensitive to this drug include $N$. asteroides, A. israelii, Clostridium spp, and C. diphtheriae. ${ }^{22}$

Fusidic acid (FA) and sodium fusidate (SF) are available in the market as cream or ointment formulation, which have low absorption and penetration through the skin layers, and do not last for a long time on the skin. Our challenge is to make a formulation with better stability, spreadability, absorption and penetration with a minimal concentration and little irritation. Our formulation is nanoemulgel.

When dealing locally with infectious diseases the percutaneous forms of antibiotics are often used. Several penetration-promoting agent ${ }^{23,24}$ and particle sizes have been studied, ${ }^{25}$ and various formulations as liposomes, ${ }^{26}$ ointments and creams ${ }^{27,28}$ and gels ${ }^{29-32}$ have been inspected and applied to improve skin penetration. Therefore, the application of nanoparticles is expected to improve the drug penetration through the skin, and local DDSs which have used it have the potential for extensive clinical use. In contrast, nanoparticle skin uptake can be affected by differences in particle size, the retention time during circulation and the possibility for adherence and degradation. ${ }^{33}$ The objective of our study is to develop topical fusidic acid and sodium fusidate nanoemulgel formulation and evaluate their antimicrobial activity.

\section{Materials and Methods Materials}

Fusidic Acid, Sodium Fusidate, Fusylin cream (Market product of Fusidic Acid) and Fusylin ointment (Market product of Sodium Fusidate) were kindly gifted to the researchers by Jerusalem Pharmaceutical company-Palestine. Tween 80, Span 20, glycerol, Tween 20, Span 80, ethanol and Carboxyvinyl polymer (Carbopol 940) were purchased from CBC Co., Ltd., Japan. Pine oil, olive oil and paraffin oil were obtained from the AL-Shams company, Palestine.

\section{Wavelength Screening for the Drugs (FA, SF) Using UV Spectrophotometer}

Scanning of the optimum wavelength for FA and SF, the samples were prepared using $10 \mathrm{~mL}$ of methanol and 0.02 $\mathrm{g}$ of the active ingredient (FA and SF). The samples were then mixed using a vortex mixer to ensure the homogeneity of the samples; the absorbance was determined using a UV spectrophotometer (Jenway 7315-spectrophotometer, United Kingdom) at a wavelength range of 200-600 nm. 


\section{Solubility Screening of FA and SF in Different Oils and Surfactants}

The solubility of the active ingredients (FA and SF) was determined by dissolving FA and SF in different oils (olive oil, paraffin oil, pine oil and corn oil) and surfactants (glycerol, Tween 20, Tween 80, Span 20 and Span 80 ) at a concentration of $2 \%$. The mixture vials were kept in an isothermal shaker (Nirmal International, Delhi, India) at $25 \pm 1.0^{\circ} \mathrm{C}$ for $72 \mathrm{~h}$ until they reached equilibrium. The supernatant of equilibrated samples was then filtered through a membrane filter $(0.22 \mu \mathrm{m})$, it was then centrifuged for $15 \mathrm{~min}$ at $3000 \mathrm{rpm}$. The absorption was determined for the drugs in each sample using a UV spectrophotometer.

\section{Preparation of FA and SF Nanoemulgel}

Fusidic acid and sodium fusidate nanoemulgel formulations were prepared, first by the preparation of FA and SF nanoemulsions, and then they were incorporated with Carbopol 940 hydrogel to produce the nanoemulgel.

\section{Preparation of Pine Oil Nanoemulsion}

The selection oil and surfactants, which were used to optimise the nanoemulsion formulation, was based on the solubility test of FA and SF in different oils and surfactants. A self-nanoemulsifying technique was used to prepare the nanoemulsion. In order to optimise the nanoemulsion formulation, different combinations of pine oil, Tween 80 and Span 20 were used to construct ternary phase diagram. Each formulation was weighed then vortexed for 2 mins, after that, it was self-emulsified in distilled water under gentle agitation to produce the nanoemulsion.

\section{Droplet Size and Polydispersity Index Analysis of Pine Oil Nanoemulsion}

The pine oil and surfactant emulsion droplet size and size distribution were determined by a laser diffraction particle and droplet size analyzer (SALD-2300, SALD-MS23, Shimadzu Corp., Japan), which allowed the measurement of the polydispersity and diameter of the droplet.

\section{Loading the Drug in Pine Oil Nanoemulsion Formulation}

The drug was loaded into the emulsion formulation after we made sure that the size was in nanoparticles, the loading process was done by dissolving FA and SF in pine oil, Span 20 and Tween 80.

\section{Hydrogel Preparation}

The hydrogel was prepared by adding Carbopol 940 to water then continuously stirring the mixture using a homogenizer to produce a uniform dispersion. The $\mathrm{pH}$ of the hydrogel was adjusted to $\mathrm{pH} 6$ using $2 \mathrm{M} \mathrm{NaOH}$, which was added by continuous stirring. The mixture was subjected to constant stirring and was left for $24 \mathrm{hrs}$ for complete gelation. ${ }^{34}$

\section{Nanoemulgel Preparation of FA and SF}

The drug loaded in the optimum nanoemulsion formulation was incorporated with Carbopol 940 hydrogel in different concentrations $(0.4 \%, 0.6 \%, 0.8 \%$ Carbopol). The obtained nanoemulgel formulations were subjected to particle size, polydispersity index (PDI) and zeta potential analysis. The particle size and PDI analysis were mentioned previously.

\section{Zeta Potential Measurement of FA and SF Nanoemulgel Formulations}

The measurement of the zeta potential was carried using Omni Brookhaven (Brookhaven Instruments Corporation, NY). The zeta potential value was measured in triplicate to calculate the average, the value of each sample was determined, and the resulting data were graphed in order to obtain a zeta potential versus Carbopol concentration.

\section{Rheological Measurement for Nanoemulgel}

There are some differences in the behavior of nanoemulgel formulations due to different concentrations of carbopol as a thickening agent; the measurement was done at the same temperature of $25^{\circ} \mathrm{C}$ by using a rotational viscometer (Brookfeild DVI, USA). The viscosity values were within the shear rate range of $0-100 \mathrm{rpm}$.

\section{Release Test of the Drug from Different Concentrations of Carbapol Using a Dialysis Membrane Bag}

Release test was performed to study the release of the drug from the nanoemulgel system using a dialysis test by adding $5 \mathrm{~g}$ of each sample (FA and SF) that contained a different concentration of carbopol $(0,4 \%, 0.6 \%, 0.8 \%$ and $1 \%$ ) in the dialysis bad (Spectra/Pro 3 Dialysis Membrane, P/N 132700) with diameter size $16 \mathrm{~mm}$. 
Phosphate buffer saline was prepared by dissolving 2.38 $\mathrm{g}$ of disodium hydrogen phosphate, $0.19 \mathrm{~g}$ of potassium dihydrogen phosphate and $8.0 \mathrm{~g}$ of sodium chloride in water, which was diluted to $1000 \mathrm{~mL}$ and the $\mathrm{pH}$ was adjusted to 2.23 . Then, the samples were added to the dialysis bag at $40 \mathrm{~mL}$ of phosphate buffer in the isothermal shaker (Nirmal International, Delhi, India) at $37 \pm 1.0^{\circ} \mathrm{C}$ to Take the samples from the buffer solution at 10, 20, 30, 60 and $120 \mathrm{~min}$ to recognize the release of the drug from the nanoemulgel. Finally, we compared the results of releasing an active ingredient (FA and SF) from the nanoemulgel samples with the market product by preforming the release test on the market product too.

\section{Anti-Bacterial Test Microorganisms}

The organisms used for the anti-bacterial test were Enterococcus fuesium (ATCC 700221), Escherichia coli (ATCC 25922), Staphylococcus aureus (ATCC 25923), Shigella sonnie (ATCC 25931), and against the growth of a diagnostically-conformed MethicillinResistant Staphylococcus aureus (MRSA), refence microbial strains were obtained from American Type Culture Collection (ATCC).

\section{Culture Media}

Moller Hinton Agar (which is produced by Becton, Dickinson and Sparks Co. in France) was used as the culture media. It contains $2 \mathrm{~g}$ of beef extract, $17.5 \mathrm{~g}$ of acid hydrolysate of casein, $1.5 \mathrm{~g}$ of starch and $17 \mathrm{~g}$ of agar per litre of purified water. The preparation of the media was done by mixing the components with each other and heating the mixture until boiling with frequent agitation to dissolve them. Then, the samples were placed in the autoclave for $20 \mathrm{mins}$ at $121^{\circ} \mathrm{C}$. After this, the agar was cooled and poured into sterile Petri dishes and on a flat surface to obtain uniform surface and depth. The samples were then stored in a refrigerator at $4-8^{\circ} \mathrm{C}$.

To study the diffusion of the Muller Hinton agar preparations: Muller Hinton agar was used for the standard inoculum of bacterial culture; $6 \mathrm{~mm}$ diameter wells were punched into the agar and three holes (A, B and C) were made. A was filled with fusidic acid or sodium fusidate nanoemulgel, B was filled with pine oil pure extract and $\mathrm{C}$ was filled with fusidic acid or sodium fusidate from the market. The plates were incubated at $37^{\circ} \mathrm{C}$ for $24 \mathrm{hrs}$. The antibacterial activity was evaluated by measuring the diameter of the zone of inhibition all tests were done in triplicate.

\section{Statistical Assessment}

All the outcomes data of the established experiments were performed in triplicate and the values were expressed as mean \pm S.D. Statistical significance was considered when the $p$ value was $\leq 0.005$.

\section{Results}

\section{Wavelength Screening for the Drugs (FA and SF) Using a UV Spectrophotometer}

The screening was carried to find an optimum and suitable wavelength for both fusidic acid and sodium fusidate using a UV spectrophotometer; an optimum absorbance was achieved at $285 \mathrm{~nm}$ for fusidic acid and $290 \mathrm{~nm}$ for Sodium fusidate.

\section{Solubility Screening of FA and SF in Different Oils and Surfactants}

The solubility of fusidic acid and sodium fusidate was determined by dissolving them in different oils and surfactants, then using a UV spectrophotometer to measure the absorption. The results obtained are explained in Table 1.

According to the results in the table above the best solubilising oil for both drugs was pine oil. In addition, Tween 80 and Span 20 showed the highest solubilising capability for the drugs among surfactants; therefore, they were chosen as surfactant and co-surfactant, respectively.

\section{Optimisation of Pine Oil Nanoemulsion}

\section{Formulations}

A self-emulsifying technique was used to prepare the pine oil nanoemulsion. The optimum nanoemulsion was chosen based on their droplet size and polydispersity index of the pine oil in the formulation. Therefore, in order to find the optimum formulation, a ternary phase diagram was constructed using different concentrations of pine oil, Tween 80 and Span 20. As presented in Figure 1, the ternary phase diagram showed a high nanoemulsion region; eight compositions showed a nanoemulsion with a droplet size below $200 \mathrm{~nm}$, as shown in Table 2. Therefore, those formulations were loaded with fusidic acid and sodium fusidate, after that their particle size and polydispersity index were measured in triplicate. 
Table I Values That Expresses the Solubility of FA and SF in Different Oils and Surfactants

\begin{tabular}{|l|l|l|}
\hline Oil & $\begin{array}{l}\text { Fusidic Acid } \\
(\mathbf{m g} / \mathbf{m L})\end{array}$ & Sodium Fusidate $(\mathbf{m g} / \mathbf{m L})$ \\
\hline Olive oil & $10.26 \pm 0.11$ & $16.72 \pm 0.28$ \\
Pine oil & $43.11 \pm 0.43$ & $42.47 \pm 0.47$ \\
Paraffin oil & $1.80 \pm 0.32$ & $5.68 \pm 0.23$ \\
Corn oil & $6.09 \pm 0.24$ & $7.97 \pm 0.20$ \\
Glycerol & $1.94 \pm 0.14$ & $4.87 \pm 0.19$ \\
Span 20 & $14.43 \pm 0.21$ & $18.98 \pm 0.27$ \\
Span 80 & $17.15 \pm 0.34$ & $17.44 \pm 0.18$ \\
Tween 20 & $7.48 \pm 0.22$ & $5.86 \pm 0.16$ \\
Tween 80 & $12.78 \pm 0.31$ & $22.97 \pm 0.41$ \\
\hline
\end{tabular}

\section{Particle Size and Polydispersibility Index of Fusidic Acid and Sodium Fusidate Nanoemulsion}

After loading the fusidic acid and sodium fusidate in the selected nanoemulsion formulations, it appears that there were variations in the particle size for both drugs with an increase in the size after loading the drugs as shown in Tables 3 and 4. Therefore, in order to choose the optimum formulation, three criteria were taken into considerations. These criteria were the drug particle size, polydispersity index and the oil concentration. From the comparison between the formulations, it can be seen that formulation eight was the selected formulation as it appears in both tables and is able to produce a formulation with the lowest particle size and a polydispersity with the highest concentration of oil at $50 \%$. The result of the fusidic acid was $140.58 \mathrm{~nm}$ in size

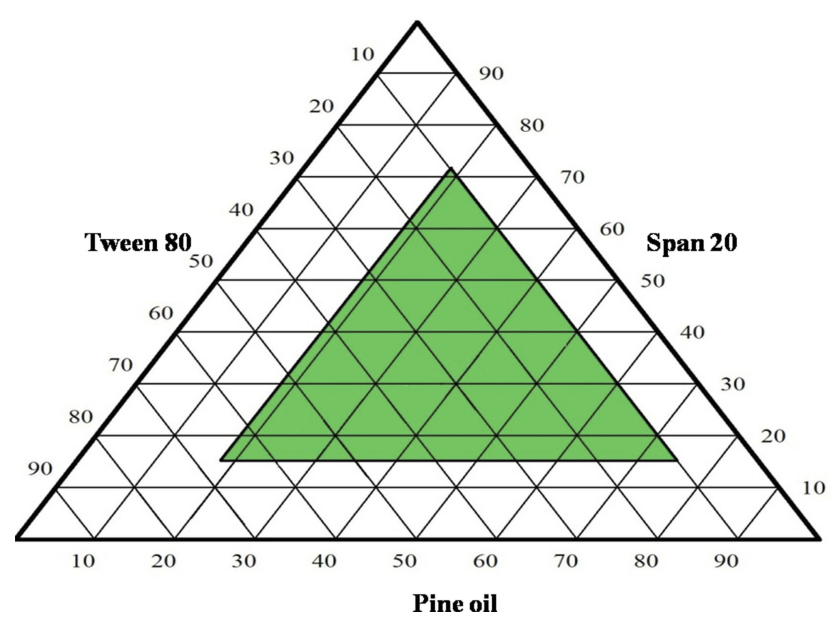

Figure I A ternary phase diagram of pine oil nanoemulsion with Tween 80 and Span 20. Data represented as mean $(n=3)$. with a polydispersity index of 0.186 , and it was $151.86 \mathrm{~nm}$ in size with a polydispersity index of 0.201 for sodium fusidate.

\section{Fusidic Acid and Sodium Fusidate Nanoemulgel Particle Size, Polydispersity Index and Zeta Potential}

Nanoemulgels of fusidic acid and sodium fusidate were prepared after loading the nanoemulsion with the drug; the mixture was then mixed with different concentrations of Carbopol hydrogel $(0.4,0.8$ and 1\%). Figures 2-4 showed the results of the particle size of the drug nanoemulgel, polydispersity index and the zeta potential, respectively. The results of the drugs particle size and PDI showed no significant difference between the drugs in the form of a nanoemulsion and when it was converted to a nanoemulgel. In addition, there were similar behaviours with respect to the difference in the Carbopol concentration; only a slight incensement appeared in the particle size with respect to the increment in the Carbopol concentration. With regard to the zeta potential, all of the nanoemulgel formulations showed a zeta potential below -30 $\mathrm{mV}$ for both drugs.

\section{Rheological Measurement for Nanoemulgel}

Both drug nanoemulgel formulations, which are considered semi-solids forms, required evaluation for their rheological properties (flow properties), which may reflect and indicate the quality and efficacy of the formulations. The rheological analysis of the drug nanoemulgel formulations showed a decrease in the viscosity with an increase in the shear rate, indicating that the drug nanoemulgel formulations behave as a pseudo-plastic (Figure 5). As it can be seen in Figure 5, the behaviour of nanoemulgel formulations was not affected by the Carbopol concentration; however, the only change was in the viscosity as it increased when with the Carbopol concentration increment.

\section{Release Test of Fusidic Acid and Sodium Fusidate from the Nanoemulgel Formulations}

The release tests of fusidic acid and sodium fusidate were carried out to study the release of the drugs from the nanoemulgel formulations, which helps in choosing the optimum Carbopol concentration used as thickening agent in producing the hydrogel, which was used in the nanoemulgel formulations. The release of the nanoemulgel formulations was compared with the release of the market product. The 
Table 2 The Selected Pine Oil Nanoemulsion Formulations

\begin{tabular}{|l|l|l|l|l|l|}
\hline Formulation & Tween 80 (\%) & Span 20 (\%) & Pine Oil (\%) & Droplets Size (nm) $\mathbf{\pm 5}$ & Polydispersibility Index $\mathbf{\pm 0 . 0 5}$ \\
\hline 1 & 0.25 & 0.55 & 0.20 & 157.18 & 0.286 \\
2 & 0.30 & 0.50 & 0.20 & 145.49 & 0.234 \\
3 & 0.50 & 0.30 & 0.20 & 155.46 & 0.321 \\
4 & 0.45 & 0.20 & 0.35 & 150.90 & 0.244 \\
5 & 0.65 & 0.15 & 0.20 & 152.59 & 0.260 \\
6 & 0.70 & 0.10 & 0.20 & 103.66 & 0.405 \\
7 & 0.60 & 0.05 & 0.35 & 128.54 & 0.263 \\
8 & 0.45 & 0.05 & 0.50 & 137.66 & 0.290 \\
\hline
\end{tabular}

Table 3 Fusidic Acid Nanoemulsion Particle Size and the Polydispersity Index

\begin{tabular}{|l|l|l|l|l|l|}
\hline Formulation & Pine Oil (\%) & Tween 80 (\%) & Span 20 (\%) & Particle Size (nm) $\mathbf{\pm 5}$ & Polydispersibility Index $\mathbf{0}$.05 \\
\hline 1 & 0.20 & 0.25 & 0.55 & 210.78 & 0.304 \\
2 & 0.20 & 0.30 & 0.50 & 331.34 & 0.156 \\
3 & 0.20 & 0.50 & 0.30 & 176.22 & 0.324 \\
4 & 0.35 & 0.45 & 0.20 & 161.65 & 0.300 \\
5 & 0.20 & 0.65 & 0.15 & 145.00 & 0.320 \\
6 & 0.20 & 0.70 & 0.10 & 209.58 & 0.551 \\
7 & 0.35 & 0.60 & 0.05 & 224.20 & 0.294 \\
8 & 0.50 & 0.45 & 0.05 & 140.58 & 0.186 \\
\hline
\end{tabular}

Table 4 Sodium Fusidate Nanoemulsion Particle Size and the Polydispersity Index

\begin{tabular}{|l|l|l|l|l|l|}
\hline Formulation & Pine Oil (\%) & Tween $\mathbf{8 0}(\%)$ & Span 20 (\%) & Particle Size (nm) $\mathbf{\pm 5}$ & Polydispersibility Index $\mathbf{0 0 . 0 5}$ \\
\hline 1 & 0.20 & 0.25 & 0.55 & 166.23 & 0.288 \\
2 & 0.20 & 0.30 & 0.50 & 170.50 & 0.368 \\
3 & 0.20 & 0.50 & 0.30 & 189.43 & 0.320 \\
4 & 0.35 & 0.45 & 0.20 & 172.42 & 0.162 \\
5 & 0.20 & 0.65 & 0.15 & 198.04 & 0.373 \\
6 & 0.20 & 0.70 & 0.10 & 167.44 & 0.298 \\
7 & 0.35 & 0.60 & 0.05 & 171.54 & 0.374 \\
8 & 0.50 & 0.45 & 0.05 & 151.67 & 0.201 \\
\hline
\end{tabular}

dialysis method was used to carry out this task. The results of the release study are explained in Figure 6. Figure 6 demonstrates the difference in the release profile of the drugs, it can be noticed that the increase in the Carbopol concentration led to a decrease in the release profile with the highest drug release at $0.4 \%$ Carbopol, in addition to this, all
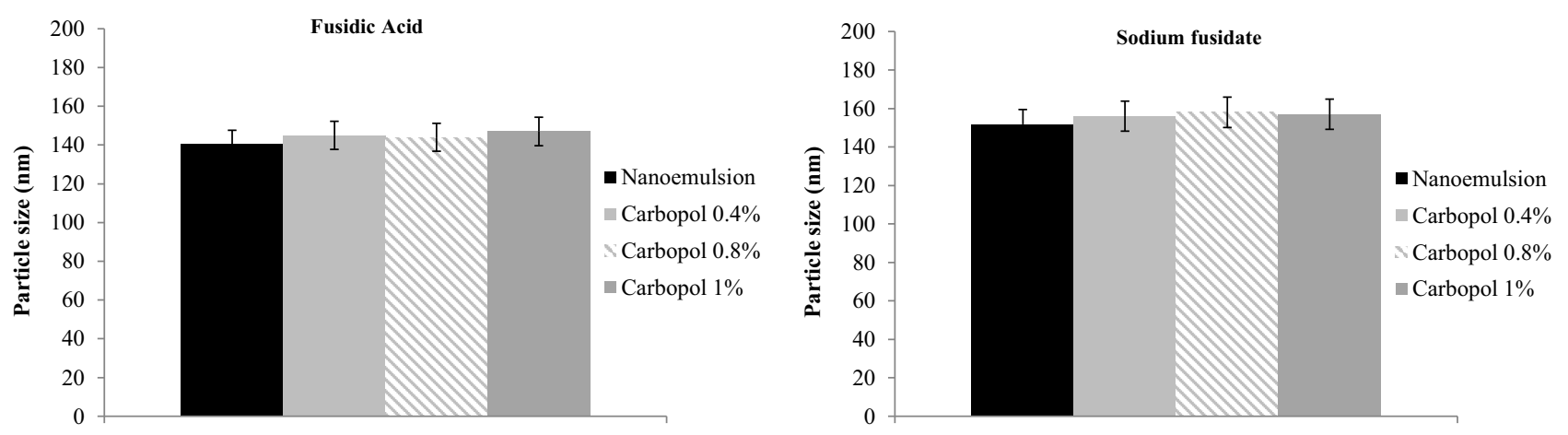

Figure 2 The particle size of fusidic acid and sodium fusidate nanoemulgels, Data represented as mean \pm SD $(n=3)$. 

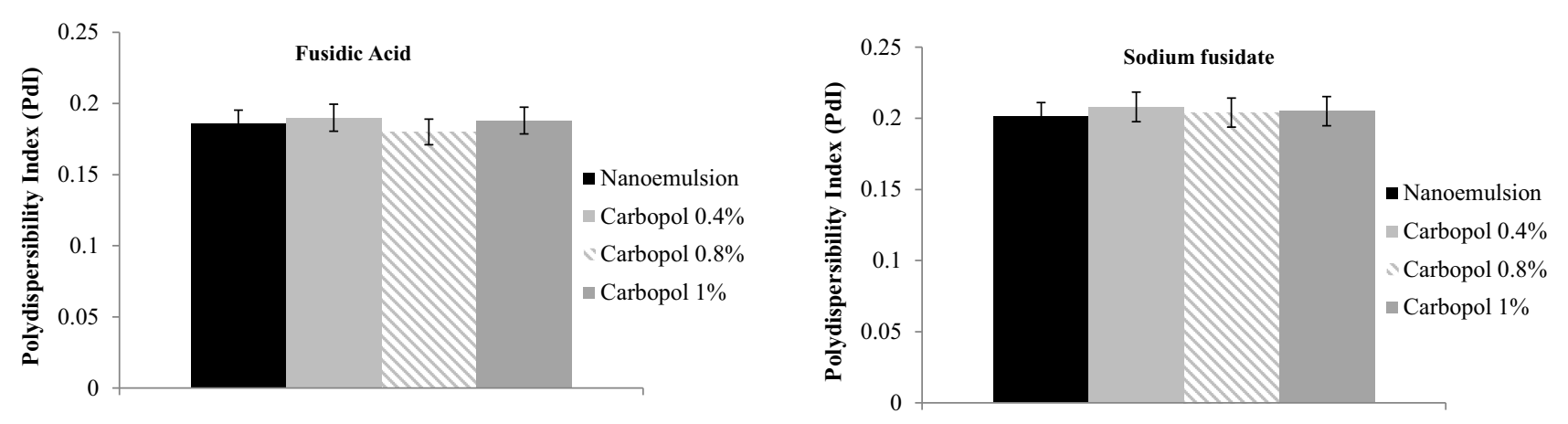

Figure 3 Polydispersity index of fusidic acid and sodium fusidate nanoemulgels, Data represented as mean \pm SD $(n=3)$.
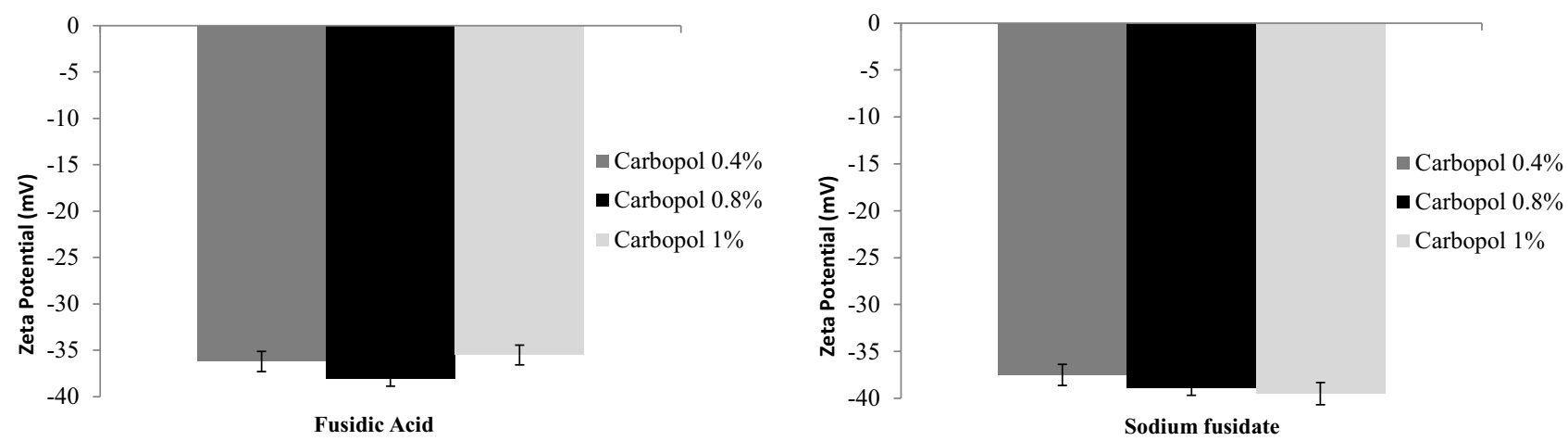

Figure 4 Zeta potential of fusidic acid and sodium fusidate nanoemulgels, Data represented as mean \pm SD $(n=3)$.

of the nanoemulgel formulations showed a higher release profile when compared with the market product.

\section{Antibacterial Evaluation of Fusidic Acid and Sodium Fusidate Nanoemulgels}

The anti-bacterial test was done on Enterococcus, Escherichia coli, Staphylococcus aureus, Shigella, and $M R S A$ by implanting them in agar media on Petri dishes to check the anti-bacterial activity of the fusidic acid and sodium fusidate nanoemulgels, this was then compared with the market by measuring the inhibition zone. Table 5 explains the anti-bacterial activity.

\section{Discussion}

Fusidic acid and sodium fusidate nanoemulgel were prepared in this study to improve their therapeutic activity when compared with the market products. To achieve this task, the drugs were prepared in a nanoemulsion form using a self-nanoemulsifying technique; this was then incorporated with Carbopol hydrogel. The drugs release and antibacterial tests were carried out for the drug nanoemulgel and the market products.
To design a nanoemulsion using a self-emulsification technique with physiochemical properties that can be accepted for the final product, the ingredient (active or inactive) of the system must be carefully chosen to have a good combination of oil, surfactant and co-surfactant. By studying the solubility, we can identify suitable selfemulsification system components with an acceptable solubilising capacity for both the drugs (FA and SF). It is important to try the technique with different oils to identify which one is more suitable and to try different cosurfactants and surfactants to have the best solubilising potential for the drugs under investigation to load the drug to achieve optimum results. ${ }^{35}$ The main excipients are the oils that can specifically solubilise the lipophilic drug due to increasing the absorption and penetration by increasing the transporting fraction. The medium chain of the oily phase of pine oil is the reason why we selected the pine oil for its amphiphilic nature, which enhanced drug solubilisation by providing it with surfactant properties. ${ }^{36}$

According to the physiochemical characteristic of pine oil, which contains linoleic acid and oleic acid which is an unsaturated medium chain fatty, ${ }^{37}$ the drugs were able to be dissolved because of the excellent solubilizing properties of 


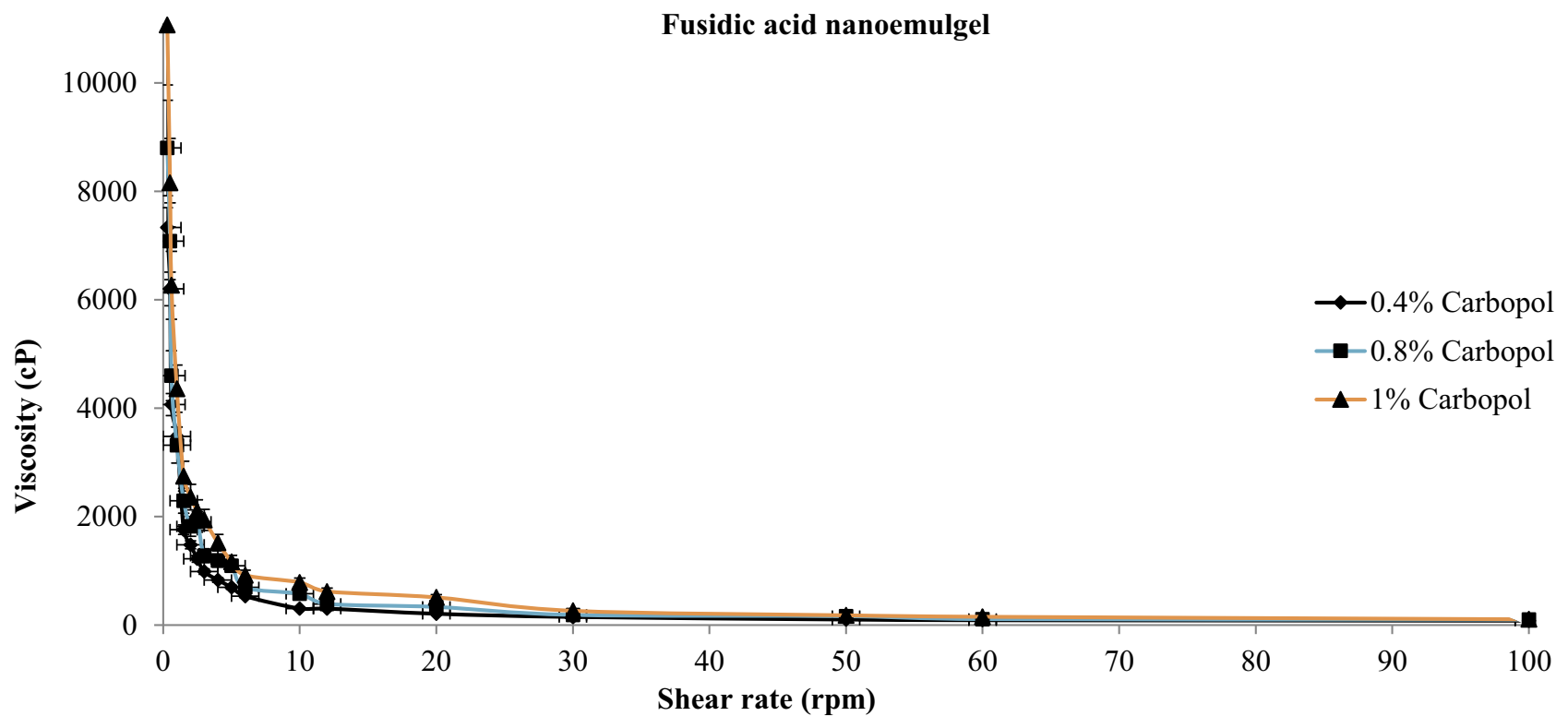

Figure 5 Rheological behaviour of fusidic acid and sodium fusidate nanoemulgels, Data represented as mean $\pm S D(n=3)$.
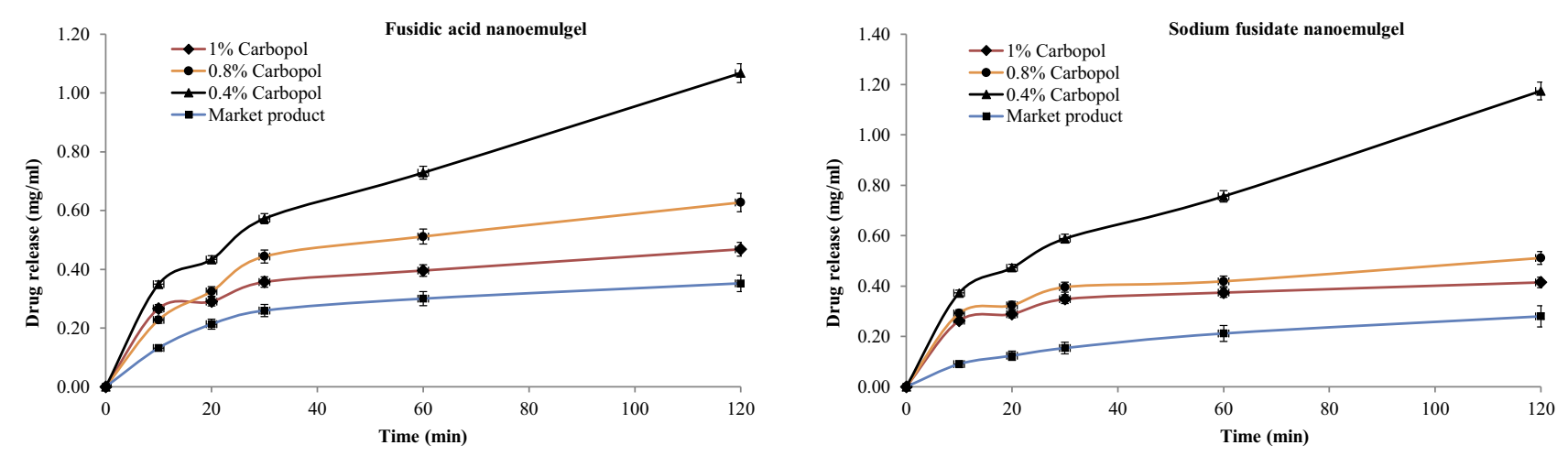

Figure 6 The release profile of fusidic acid and sodium fusidate nanoemulgels, Data represented as mean \pm SD $(n=3)$.

the oil as it is composed of fatty acids which help in developing a formulation containing the drug.

The surfactants used were hydrophilic (non-ionic), safe and biocompatible. ${ }^{38}$ Also, they formed superior and uniform droplets, which allowed the nanoemulsion to have a rapid drug release and rapid absorption due to a large surface area. The hydrophilic-lipophilic balance (HLB) value of the surfactant Tween 80 is 15, and the HLB value of co-surfactant Span 20 is 8.6, to have a system with high HLB value more than 10 by combining them to get an olw emulsion easily. ${ }^{39}$

Table 5 Nanoemulgel Activity Against Enterococcus

\begin{tabular}{|c|c|c|c|c|c|}
\hline Bacteria & $\begin{array}{l}\text { Sodium Fusidate } \\
\text { Nanoemulgel }\end{array}$ & $\begin{array}{l}\text { Market Sodium } \\
\text { Fusidate }\end{array}$ & $\begin{array}{l}\text { Fusidic Acid } \\
\text { Nanoemulgel }\end{array}$ & $\begin{array}{l}\text { Market Fusidic } \\
\text { Acid }\end{array}$ & Pine Oil \\
\hline Enterococcus & $3.5 \pm 0.1 \mathrm{~cm}$ & $2 \pm 0.2 \mathrm{~cm}$ & $4.5 \pm 0.4 \mathrm{~cm}$ & $3 \mathrm{~cm}$ & $1.7 \pm 0.3 \mathrm{~cm}$ \\
\hline E. coli & $1.4 \pm 0.2 \mathrm{~cm}$ & Negative & $1.4 \pm 0.1 \mathrm{~cm}$ & Negative & $\mathrm{I} \pm 0.1 \mathrm{~cm}$ \\
\hline $\begin{array}{l}\text { Staphylococcus } \\
\text { aureus }\end{array}$ & $7 \pm 0.2 \mathrm{~cm}$ & $4 \pm 0.1 \mathrm{~cm}$ & $6.8 \pm 0.2 \mathrm{~cm}$ & $4 \pm 0.1 \mathrm{~cm}$ & $3 \pm 0.3 \mathrm{~cm}$ \\
\hline Shigella & $4.5 \pm 0.3 \mathrm{~cm}$ & $0.5 \pm 0.2 \mathrm{~cm}$ & $4.6 \pm 0.5 \mathrm{~cm}$ & $\mathrm{I} \pm 0.4 \mathrm{~cm}$ & $2 \pm 0.5 \mathrm{~cm}$ \\
\hline MRSA & $5.8 \pm 0.5 \mathrm{~cm}$ & $3 \pm 0.3 \mathrm{~cm}$ & $6.4 \pm 0.2 \mathrm{~cm}$ & $3.4 \pm 0.5 \mathrm{~cm}$ & $2.3 \pm 0.2 \mathrm{~cm}$ \\
\hline
\end{tabular}


Construction a ternary phase diagram to determine the self-nanoemulsifying regions and to select appropriate concentrations of oil, surfactant and co-surfactant used in the formulation of the optimum self-nanoemulsifying drug delivery system (SNEDDS) formulation. The diagram was plotted to show the best formulation that could be obtained with a droplet size below $200 \mathrm{~nm}$. Obtaining a high nanoemulsion area of pine oil was possible due to the optimum balance of the solubility, HLB values and molecular geometry; this was confirmed by Mayer and his research team. ${ }^{40}$

The droplet size was a critical factor in the SNEEDS performance due to identifying the extent and rate of the drug release and drug absorption. In addition, the smaller particle size, the larger interfacial surface area led to further rapid absorption and improvement of the bioavailability. When the droplet size was lower than $200 \mathrm{~nm}$, it led to achieving the criteria of SNEDDS. The proper mixture of surfactantlco-surfactant provided a smaller globule size, a strong mechanical barrier that protected the formed globule to be aggregated as clarified. ${ }^{41}$ The polydispersity index (PDI) is another parameter of equal importance to the droplet size in the formulation of SNEDDS; It is also called the droplet size distribution to measure the particle homogeneity. The particles are more homogenous when they are close to zero of the PDI value. Small values indicate a narrow globule size distribution, homogeneity and more uniform emulsion which has higher physical stability.

The particle size and PDI of the drugs showed no significant difference between them in the form of nanoemulsion and when it was converted to nanoemulgel. The indication of the stability of the colloidal preparation is referred to as the magnitude of the zeta potential. If the zeta potential of the particles is largely negative or positive, the particles will repel each other making dispersion stable, and if the values are low, there is a dispersion instability because there is no force preventing them from coming together. There is a dividing line that separates between stable and unstable dispersions which is generally determined at +30 or $-30 \mathrm{mV}^{42}$ When the zeta potential of the particles was higher than $+30 \mathrm{mV}$ or lower than $-30 \mathrm{mV}$ it was typically considered stable. All of the nanoemulgel formulations showed a zeta potential between -35 to $-40 \mathrm{mV}$ for both the drugs due to the presence of non-ionic surfactants, which stabilise the system by making a coat around their surface. They do not contribute to any change in the nanoemulsion particle, so it does not affect the stability of producing a nanoemulsion. ${ }^{43}$

The rheological measurements are important for semisolid preparations to describe the system physically (flow properties) and to control the stability, which is important in ensuring the effectiveness and quality of the formulation. Viscosity is a crucial factor as higher viscosities may affect drug release and bioavailability due to the slowing down in the drug diffusion out of the vehicle. ${ }^{44}$ From the results, the viscosity increased when the Carbopol concentration increased; this also decreased the shear rate. The rheological behaviour of the colloidal-emulgel was pseudo-plastic, ie, the viscosity decreases with an increase in the shear rate.

The fast release of the drug into the dissolution medium describes the efficiency of the delivery system. ${ }^{39,45}$ The result of the drug release correlates with the concentration of Carbopol; it will decrease in every increment of Carbopol due to the viscosity that the Carbopol confers on it. Therefore, the formulation that contains $0.4 \%$ Carbopol will have the best pattern of drug release relative to other formulations in the study $(0.8 \%$ and $1 \%)$ and the market product. These results were proven by many studies as stated by Sanjay Kumar et al (2018), who found that the increase in the Carbopol (sustained releasing agent) concentration affected metoprolol succinate release from the dosage form. ${ }^{46}$

Fusidic acid and sodium fusidate nanoemulgels showed an improvement and higher zone of inhibition in their antibacterial activity when compared to the market drug. These results are due to several factors. First, as the nanoparticles have a small nano-size and a larger surface, this leads to higher penetrations and activity. ${ }^{47}$ The same finding was mentioned by Marslin et al (2015), who studied the effect of Withania somnifera cream incorporated with silver nanoparticles on the microbial growth. They found an increase in the penetration of Withania somnifera cream silver nanoparticles, which led to an increase in the inhibition of the bacterial growth. In addition, the packing process of the bacteria by nanoparticles increased the concentration of the drugs entering the bacteria by increasing the contact between the drug and the bacteria. ${ }^{48}$ The same results were obtained in 2017 by Assali et al who reported an improvement in the antimicrobial activity of ciprofloxacin that contains single-walled carbon nanotubes which led to an increase in the residence time of the drug by the aggregation of it around the bacteria as well as leading to more penetration through it. ${ }^{49}$ 
The second reason for the improvement in the bacterial inhibition was due to the presence of pine oil, which showed anti-microbial activity against gram-negative and gram-positive bacteria and fungi, which led to the enhancement of the drug nanoemulgels. Recent research has shown that pine oil has antimicrobial activity against Staphylococcus aureus (which is a bacterial pathogen that is the main cause for skin infections), MRSA, E. coli or Listeria monocytogenes, Pseudomonas aragenusaand $\alpha$ pinene and terpineol. The antibacterial activity of pine oil was referred to by the presence of essential oils asphellandrene, terpineol and mainly terpinen-4-ol for S. aureus $^{50}$ and $\beta$-caryophyllene, which is responsible for destroying cellular integrity, inhibition of respiration and ion transportation for $\mathrm{B}$. cereus according to the study of depa in $2008 .^{51}$

\section{Conclusion}

In this paper, fusidic acid and sodium fusidate nanoemulgels were prepared successfully by the development of a nanoemulsion, which was composed of pine oil, Tween 80 and Span 20. The nanoemulsion was prepared by a selfnanoemulsifying technique, which was then incorporated with Carbopol hydrogel. These nanoemulgels were found to have high stability indicated by the zeta potential, a similar rheological behaviour, in addition to this, the highest drug release and antimicrobial activity was found to be at $0.4 \%$ Carbopol when compared to the market product. In general, the results of the study directed that fusidic acid and sodium fusidate nanoemulgels reported in this study could be promising dosage forms for pharmaceutical industries. The findings reported in this paper will also be a guide towards the use of simple nanotechnology techniques in the preparation of pharmaceutical dosage forms.

\section{Acknowledgements}

Sincere thanks to all participants in the study are also acknowledged.

\section{Disclosure}

The authors report no conflicts of interest in this work.

\section{References}

1. DeLouise LA. Applications of nanotechnology in dermatology. J Invest Dermatol. 2012;132(3):964-975. doi:10.1038/jid.2011.425

2. Hirose A, Nishimura T, Kanno J. Research strategy for evaluation methods of the manufactured nanomaterials in NIHS and importance of the chronic health effects studies. Bull Nati Inst Health Sci. 2009;127:15-25.
3. Saini R, Saini S, Sharma S. Nanotechnology: the future medicine. $J$ Cutan Aesthet Surg. 2010;3(1):32-33. doi:10.4103/09742077.63301

4. Chellapa P, Eid AM, Elmarzugi NA. Preparation and characterization of virgin coconut oil nanoemulgel. J Chem Pharm Res. 2015;7 (9):787-793.

5. Sutradhar KB, Amin ML. Nanoemulsions: increasing possibilities in drug delivery. Eur J Nanomed. 2013;5(2):97-110. doi:10.1515/ejnm2013-0001

6. Baibhav J, Gurpreet S, Rana A, et al. Development and characterization of clarithromycin emulgel for topical delivery. Int J Drug Dev Res. 2012;4(3):310-323.

7. Vikas S, Seema S, Gurpreet S, et al. Penetration enhancers: a novel strategy for enhancing transdermal drug delivery. Int Res J Pharm. 2011;2:32-36.

8. Desai P, Mhaskar GM. Formulation and evaluation of Zingiber officinale Emulgel. Int J Pharm Sci Drug Res. 2019;12(3):1294-1300.

9. Singla V, Saini S, Joshi B, et al. Emulgel: a new platform for topical drug delivery. Int J Pharma Bio Sci. 2012;3(1):485-498.

10. Michael CA, Dominey-Howes D, Labbate M. The antimicrobial resistance crisis: causes, consequences, and management. Public Health Front. 2014;2:145. doi:10.3389/fpubh.2014.00145

11. Aruguete DM, Kim B, Hochella MF, et al. Antimicrobial nanotechnology: its potential for the effective management of microbial drug resistance and implications for research needs in microbial nanotoxicology. Environ Sci Proc Imp. 2013;15(1):93-102.

12. Baptista PV, McCusker MP, Carvalho A, et al. Nano-strategies to fight multidrug resistant bacteria-“A battle of the titans". Front Microbiol. 2018;9:1441. doi:10.3389/fmicb.2018.01441

13. Godtfredsen W, Jahnsen S, Lorck H, et al. Fusidic acid: a new antibiotic. Nature. 1962;193:987. doi:10.1038/193987a0

14. Turnidge J. Fusidic acid pharmacology, pharmacokinetics and pharmacodynamics. Int $J$ Antimicrob Agents. 1999;12:S23-S34. doi:10.1016/S0924-8579(98)00071-5

15. Fuller FW. The side effects of silver sulfadiazine. J Burn Care Res. 2009;30(3):464-470. doi:10.1097/BCR.0b013e3181a28c9b

16. Lipsky BA, Hoey C. Topical antimicrobial therapy for treating chronic wounds. Clin Infect Dis. 2009;49(10):1541-1549. doi:10.1086/599193

17. Fisher A. Lasers and allergic contact dermatitis to topical antibiotics, with particular reference to bacitracin. Cutis. 1996;58(4):252-254.

18. Levy SB, Marshall B. Antibacterial resistance worldwide: causes, challenges and responses. Nat Med. 2004;10(12):122-129. doi: $10.1038 / \mathrm{nm} 1145$

19. Katare OP, Raza K, Singh B, et al. Novel drug delivery systems in topical treatment of psoriasis: rigors and vigors. Indian J Dermatol Ve. 2010;76(6):612-621. doi:10.4103/0378-6323.72451

20. Vanangamudi SS, Srinivasan M, Chulliel NN, et al. Novel Dermaceutical Cream Made Using Sodium Fusidate. Google Patents. 2011.

21. Fernandes P. Fusidic acid: a bacterial elongation factor inhibitor for the oral treatment of acute and chronic staphylococcal infections. Cold Spring Harb Perspect Med. 2016;6(1):a025437. doi:10.1101/ cshperspect.a025437

22. Ball A, Gray J, Murdoch JM. Fusidic Acid (Sodium Fusidate). In: Antibacterial Drugs Today. Dordrecht: Springer; 1978:46-48.

23. Sakeena M, Muthanna F, Ghassan Z, et al. Effect of limonene on permeation enhancement of ketoprofen in palm oil esters nanoemulsion. $J$ Oleo Sci. 2010;59(7):395-400. doi:10.5650/ jos. 59.395

24. Shah PP, Desai PR, Singh M. Effect of oleic acid modified polymeric bilayered nanoparticles on percutaneous delivery of spantide II and ketoprofen. J Control Release. 2012;158(2):336-345. doi:10.1016/j. jconrel.2011.11.016

25. Moribe K, Shibata M, Furuishi T, et al. Effect of particle size on skin permeation and retention of piroxicam in aqueous suspension. Chem Pharm Bull. 2010;58(8):1096-1099. doi:10.1248/cpb.58.1096 
26. Maestrelli F, González-Rodríguez ML, Rabasco AM, et al Preparation and characterisation of liposomes encapsulating ketoprofen-cyclodextrin complexes for transdermal drug delivery. Int J Pharm. 2005;298(1):55-67. doi:10.1016/j.ijpharm.2005.03.033

27. Bansod SD, Bawaskar MS, Gade AK, et al. Development of shampoo, soap and ointment formulated by green synthesised silver nanoparticles functionalised with antimicrobial plants oils in veterinary dermatology: treatment and prevention strategies. IET Nanobiotechnol. 2015;9(4):165-171. doi:10.1049/iet-nbt.2014.0042

28. Gürol Z, Hekimoğlu S, Demirdamar R, et al. Percutaneous absorption of ketoprofen. I. In vitro release and percutaneous absorption of ketoprofen from different ointment bases. Pharm Acta Helv. 1996;71(3):205-212. doi:10.1016/0031-6865(96)00011-8

29. Beetge E, du Plessis J, Müller DG, et al. The influence of the physicochemical characteristics and pharmacokinetic properties of selected NSAID's on their transdermal absorption. Int J Pharm. 2000;193(2):261-264. doi:10.1016/S0378-5173(99)00340-3

30. Goosen C, Du Plessis J, Müller D, et al. Correlation between physicochemical characteristics, pharmacokinetic properties and transdermal absorption of NSAID's. Int J Pharm. 1998;163(1-2):203-209. doi:10.1016/S0378-5173(97)00359-1

31. Nagai N, Ito Y. Therapeutic effects of gel ointments containing tranilast nanoparticles on paw edema in adjuvant-induced arthritis rats. Biol Pharm Bull. 2014;37(1):96-104. doi:10.1248/bpb.b1300630

32. Nagai N, Yoshioka C, Ito Y. Topical therapies for rheumatoid arthritis by gel ointments containing indomethacin nanoparticles in adjuvant-induced arthritis rat. J Oleo Sci. 2015;64(3):337-346. doi:10.5650/jos.ess 14170

33. Labouta HI, Schneider M. Interaction of inorganic nanoparticles with the skin barrier: current status and critical review. Nanomed Nanotechnol Bio Med. 2013;9(1):39-54. doi:10.1016/j.nano.2012. 04.004

34. Khurana S, Jain N, Bedi P. Nanoemulsion based gel for transdermal delivery of meloxicam: physico-chemical, mechanistic investigation Life Sci. 2013;92(6-7):383-392. doi:10.1016/j.lfs.2013.01.005

35. Humberstone AJ, Charman WN. Lipid-based vehicles for the oral delivery of poorly water soluble drugs. Adv Drug Deliv Rev. 1997;25 (1):103-128. doi:10.1016/S0169-409X(96)00494-2

36. Balakrishnan P, Lee B-J, Oh DH, et al. Enhanced oral bioavailability of dexibuprofen by a novel solid self-emulsifying drug delivery system (SEDDS). Eur J Pharm Biopharm. 2009;72(3):539-545. doi:10.1016/j.ejpb.2009.03.001

37. Vanhanen LP, Savage GP, Hider R. Fatty acid profile of New Zealand grown edible pine nuts (Pinus spp.). Food Nutr Sci. 2017;8:305-315.

38. Azeem A, Rizwan M, Ahmad FJ, et al. Nanoemulsion components screening and selection: a technical note. AAPS PharmSciTech. 2009;10(1):69-76. doi:10.1208/s12249-008-9178-x
39. Sharma S, Sharma A, Naseer M, et al. Formulation and evaluation of self emulsifying drug delivery system of ibuprofen using castor oil. Int J Pharm Pharm Sci. 2011;3:299-302.

40. Mayer S, Weiss J, McClements DJ. Vitamin E-enriched nanoemulsions formed by emulsion phase inversion: factors influencing droplet size and stability. $J$ Colloid Interface Sc. 2013;402:122-130. doi:10.1016/j.jcis.2013.04.016

41. Nepal PR, Han H-K, Choi H-K. Preparation and in vitro-in vivo evaluation of Witepsol ${ }^{\circledR} \mathrm{H} 35$ based self-nanoemulsifying drug delivery systems (SNEDDS) of coenzyme Q10. Eur J Pharm Sci. 2010;39 (4):224-232. doi:10.1016/j.ejps.2009.12.004

42. Arriaga LR, Drenckhan W, Salonen A, et al. On the long-term stability of foams stabilised by mixtures of nano-particles and oppositely charged short chain surfactants. Soft Matter. 2012;8 (43):11085-11097. doi:10.1039/c2sm26461g

43. Salim N, Basri M, Rahman MA, et al. Phase behaviour, formation and characterization of palm-based esters nanoemulsion formulation containing ibuprofen. J Nanomedic Nanotechnol. 2011;2(4):1-5. doi:10.4172/2157-7439.1000113

44. Boddupalli BM, Mohammed ZN, Nath RA, et al. Mucoadhesive drug delivery system: an overview. J Adv Pharm Tech Res. 2010;1 (4):381-387. doi:10.4103/0110-5558.76436

45. Saritha D, Bose P, Nagaraju R. Formulation and evaluation of self emulsifying drug delivery system (SEDDS) of Ibuprofen. Int J Pharm Sci Res. 2014;5:3511-3519.

46. Sahu SSK, Kumar A. Influence of carbopol and polyox on the release of metoprolol succinate from extended-release matrix tablets: a doe approach and in-vivo evaluation. World J Pharm Pharm Sci. 2018;7 (4):1228-1239.

47. Zhang L, Pornpattananangkul D, Hu C-M, et al. Development of nanoparticles for antimicrobial drug delivery. Curr Med Chem. 2010;17(6):585-594. doi:10.2174/092986710790416290

48. Marslin G, Selvakesavan RK, Franklin G, et al. Antimicrobial activity of cream incorporated with silver nanoparticles biosynthesized from Withania somnifera. Int J Nanomedicine. 2015;10:5955-5963. doi:10.2147/IJN.S81271

49. Assali M, Zaid AN, Abdallah F, et al. Single-walled carbon nanotubes-ciprofloxacin nanoantibiotic: strategy to improve ciprofloxacin antibacterial activity. Int $J$ Nanomedicine. 2017;12:6647-6659. doi:10.2147/IJN

50. Edris AE. Pharmaceutical and therapeutic potentials of essential oils and their individual volatile constituents: a review. Phytother Res. 2007;21(4):308-323. doi:10.1002/(ISSN)1099-1573

51. Deba F, Xuan TD, Yasuda M, et al. Chemical composition and antioxidant, antibacterial and antifungal activities of the essential oils from Bidens pilosa Linn. var. Radiata. Food Control. 2008;19 (4):346-352. doi:10.1016/j.foodcont.2007.04.011
International Journal of Nanomedicine

\section{Publish your work in this journal}

The International Journal of Nanomedicine is an international, peerreviewed journal focusing on the application of nanotechnology in diagnostics, therapeutics, and drug delivery systems throughout the biomedical field. This journal is indexed on PubMed Central, MedLine, CAS, SciSearch ${ }^{\mathbb{R}}$, Current Contents ${ }^{\mathbb{B}} /$ Clinical Medicine,
Journal Citation Reports/Science Edition, EMBase, Scopus and the Elsevier Bibliographic databases. The manuscript management system is completely online and includes a very quick and fair peer-review system, which is all easy to use. Visit http://www.dovepress.com/ testimonials.php to read real quotes from published authors. 